\title{
Gravitational Waves from Global Second Order Phase Transitions
}

\author{
John T. Giblin, Jr ${ }^{1,2,3}$, Larry R. Price ${ }^{4,5}$, Xavier Siemens ${ }^{4}$, and Brian Vlcek ${ }^{4}$ \\ ${ }^{1}$ Department of Physics, Kenyon College, Gambier, OH 43022 \\ ${ }^{2}$ Perimeter Institute for Theoretical Physics, 31 Caroline St. N, Waterloo, ON N2L $2 Y 5$ \\ ${ }^{3}$ Department of Physics, Case Western Reserve University, Cleveland, OH 44106 \\ ${ }^{4}$ Center for Gravitation and Cosmology, Department of Physics, \\ University of Wisconsin-Milwaukee, P.O. Box 413, Milwaukee, WI 53201 and \\ ${ }^{5}$ LIGO - California Institute of Technology, Pasadena, CA 91125, USA
}

(Dated: October 16, 2018)

\begin{abstract}
Global second-order phase transitions are expected to produce scale-invariant gravitational wave spectra. In this manuscript we explore the dynamics of a symmetry-breaking phase transition using lattice simulations. We explicitly calculate the stochastic gravitational wave background produced during the transition and subsequent self-ordering phase. We comment on this signal as it compares to the scale-invariant spectrum produced during inflation.
\end{abstract}

\section{INTRODUCTION}

A scale-invariant spectrum of gravitational radiation is a key prediction of inflation [1, 2]. Measuring the ratio of the amplitude of gravitational radiation to the amplitude of density perturbations, $r$, would be a direct probe of the inflationary energy scale. Such a measurement has eluded observation in the cosmic microwave background (CMB) thus far [3] and represents one of the key goals of future CMB observational missions [4].

On the other hand, some authors have argued that phase transitions can mimic the scale-invariant inflationary signal [5-7]. The mechanism through which this is accomplished is not trivial. The phase transition itself is not the source. Rather, energy is deposited into gravitational radiation via the self-ordering of fields as regions of spacetime become causally connected.

The process begins with $\mathcal{N}$ scalar fields, $\phi_{i}$, subject to a temperature dependent potential, $V$. At high temperatures the potential looks quadratic with a single minimum at the origin. This state has $O(\mathcal{N})$ symmetry since the state is symmetric any any rotation in the $\mathcal{N}$ dimensional field space. Once the potential drops below a critical temperature, the field acquires a vacuum expectation value (VEV). This process spontaneously breaks the $O(\mathcal{N})$ to a $O(\mathcal{N}-1)$ symmetry. The transition can be very fast, with a duration, $\tau$, that is much smaller than the Hubble time, $\tau \ll H^{-1}$. The field configuration of each Hubble volume will lie somewhere in the vacuum state, however each causally disconnected volume will be independent of the others. This process can be quite smooth, and there is no a priori reason to believe such a process radiates. However, if the Universe is radiation (and later matter) dominated after the transition, the growth of the comoving Hubble scale continuously brings previously causally disconnected regions into contact. Regions thus acquire field gradients on the scale of the Hubble horizon. These gradients generate anisotropic stresses that source gravitational waves. The growth of the horizon acts as a high-pass filter on the spectrum of gravitational waves, freezing out large-wavelength modes until they enter the horizon and become dynamical. As the horizon grows power is distributed at all scales between the physical Horizon size at the time of the transition, $H_{c}^{-1}$, and the Horizon size at the time of observation.

In all previous studies, authors have relied on large$\mathcal{N}$ approximations in order to calculate the gravitational wave signal. Here we make no such approximation and approach the problem numerically. We place $\mathcal{N}$ scalar fields on a lattice evolving in a Friedmann-LemaîtreRobertson-Walker background. Each lattice point is initialized with a field value derived from thermal initial conditions. If we allow the lattice spacing to be $H_{0}^{-1}$ at the beginning of the simulation each lattice point will settle into an independent position in the vacuum manifold. As the simulation progresses, the fields will evolve and align themselves. This self-ordering produces anisotropic stresses that source gravitational waves.

This paper is organized as follows. In Section II we will introduce a toy model of spontaneous symmetry breaking. We outline our computational methods in Section III and present the spectra we calculate in our simulations. In Section IV we will comment on the differences between the spectra we predict from self-ordering and the spectrum produced during inflation.

\section{GLOBAL SYMMETRY BREAKING}

The GUT scale naturally arises from particle physics as the scale at which the electroweak and strong couplings are of the same order of magnitude. It is likely that these forces are unified under some larger symmetry above the GUT scale and that this symmetry is broken as the Universe cools. Nevertheless, the nature of the GUT symmetry and how it is broken remains unknown.

The transition could be first-order, in which case bubbles of the broken phase nucleate and coalesce. In this model the phase transition happens very rapidly - the entirety of the universe can end up in a unique state in less than a Hubble time. This process is likely to produce gravitational radiation [8-11] as bubbles collide and coalesce. 
Conversely, the phase transition could be second-order. In this case the field smoothly transitions to the broken phase as the temperature of the universe drops. If the broken phase is not unique, that is if the vacuum state has some symmetry with respect to the field configuration, the effects of the existence of this phase transition can lead to observational effects for many Hubble times.

We begin with two assumptions: (1) that the Universe is radiation dominated at the time when the phase transition occurs, and (2) that the energy associated with the fields undergoing the phase transition is some small fraction, $\alpha$, of the total energy density at the time of the transition, $\rho_{c}$. The total density, at any time, is

$$
\rho=\rho_{\mathrm{rad}}+\rho_{\phi}
$$

where

$$
\rho_{\phi}=\sum_{i} \frac{1}{2}\left(\dot{\phi}^{2}+\frac{\left(\nabla \phi_{i}\right)^{2}}{a^{2}}\right)+V\left(\phi_{i}, T\right)
$$

and

$$
\rho_{\mathrm{rad}}=(1-\alpha) a^{-4} \rho
$$

Since the Universe is necessarily dominated by the radiation energy-density, we will only consider cases where $\alpha \ll 1$ so that the Universe is well described by assuming $H \propto a^{-4}$.

The potential in Eq. (2) is temperature dependent. To leading order in temperature,

$$
V\left(\phi_{i}, T\right)=m_{\mathrm{eff}}^{2}(T) \phi^{2}+\frac{\lambda}{8}\left(\phi^{4}+\frac{v^{4}}{4}\right),
$$

where $\phi^{2}=\sum_{i} \phi_{i}^{2}$. The temperature dependent effective mass can be parameterized by

$$
m_{\mathrm{eff}}^{2}=\frac{\lambda v^{2}}{8}\left(\frac{T}{T_{c}}-1\right) .
$$

At temperatures higher than the critical temperature, $T_{c}$, the effective mass is positive, the potential has a unique minimum at the origin, and this minimum has full $O(\mathcal{N})$ symmetry, and at the origin

$$
\left.m_{\mathrm{eff}}^{2}\right|_{\phi=0}=-\frac{\lambda v^{2}}{8} .
$$

After the phase transition the potential has an $O(\mathcal{N}-1)$ symmetric VEV

$$
V\left(\phi_{i}\right)=V\left(\phi_{i}, 0\right)=\frac{\lambda}{8}\left(\phi^{2}-\frac{v^{2}}{2}\right)^{2},
$$

The phase transition occurs at the critical temperature, $T_{c}$, when the effective mass of the field vanishes. Although the field has a mean value, $\phi=0$, there is a variance associated with this value,

$$
\sigma^{2}=\left\langle\phi^{2}\right\rangle-\langle\phi\rangle^{2}=\left\langle\phi^{2}\right\rangle,
$$

that sets the distribution of field values at the time of the transition. We can assume that at this time, each Hubble volume (sphere of radius $H_{c}^{-1}$ ) has a homogeneous field value, drawn from a Gaussian distribution (see Appendix A)

$$
P(\phi)=\sqrt{\frac{1}{2 \pi \sigma^{2}}} e^{-\frac{\phi^{2}}{2 \sigma^{2}}}
$$

and

$$
\begin{aligned}
\sigma^{2} & =\frac{T_{c}}{2 \pi^{2}} \int_{0}^{\infty} \frac{k^{2} e^{-k^{2} / H_{c}^{2}}}{k^{2}+m_{\mathrm{eff}}^{2}} d k \\
& =\frac{H_{c} T_{c}}{4 \pi^{3 / 2}}
\end{aligned}
$$

where the second equality comes from setting $m_{\mathrm{eff}}=0$.

The temperature of the Universe at the beginning of the simulation, $T_{c}$, is related to the energy density of the Universe at that time,

$$
\rho_{c}=\frac{\pi^{2}}{30} g_{c} T_{c}^{4},
$$

where $g_{c}$ is the number of ultra-relativistic degrees of freedom at the time of the phase transition. We take $g_{c}=1000$.

The average energy density in the field is well approximated by setting $\phi=0$ in Eq. (7),

$$
\left\langle\rho_{\phi}\right\rangle \approx \frac{\lambda v^{4}}{32}
$$

which is some fraction, $\alpha$, of the total energy density

$$
\frac{\lambda v^{4}}{32}=\alpha \rho_{c}=\alpha \frac{3 m_{\mathrm{pl}}^{2}}{8 \pi} H_{c}^{2} .
$$

This constrains the value of the VEV,

$$
\frac{v^{2}}{2}=\sqrt{\frac{3 \alpha}{\lambda \pi}} H_{c} m_{\mathrm{pl}} .
$$

This provides us with a good self-consistency check, namely, that the variance of the fluctuations of the filed are small compared to the $\mathrm{VEV}, v / \sqrt{2}$. This ratio is given by

$$
\frac{\sigma^{2}}{v^{2} / 2}=\frac{T_{0}}{m_{\mathrm{pl}}} \frac{1}{4 \pi} \sqrt{\frac{\lambda}{3 \alpha}},
$$

which is, in general, less that one if $T_{0}$ is significantly below $10^{19} \mathrm{GeV}$ and $\alpha$ and $\lambda$ are of similar order.

\section{GRAVITATIONAL WAVES}

Evolving scalar fields on a discrete lattice is now a mature field of study. Scalar fields were first introduced to the lattice, in a Cosmological context, by LATTICEEASY [12] and later by DEFROST [13] to study the 
non-linear dynamics of preheating after inflation. More recently the authors of [14] re-framed the question by moving the fundamental description of the fields from configuration space to momentum space. Even more recently the author of [15] introduced a versatile code that allows the user more control over the integrating scheme. Here we chose to use LATticEEASY since we are interested in sub- and super-horizon scales, large lattices with efficient storage and a specific associated potential, Eq. (7). This software natively evolves scalar fields according to the Klein-Gordon equation in an expanding background,

$$
\ddot{\phi}_{i}+3 H \dot{\phi}_{i}-\frac{\nabla^{2} \phi_{i}}{a^{2}}+\frac{\partial V\left(\phi_{i}\right)}{\partial \phi}=0,
$$

where we work in units where $c=\hbar=1$. The homogeneous background evolution is determined by

$$
H^{2}=\frac{8 \pi}{3 m_{p l}^{2}} \rho,
$$

where $\rho=\rho(t)$ is the homogeneous, average, energy density at time $t$. We couple LATTICEEASY to a code that evolves the metric perturbation using the methods of $[16,17]$.

Since the lattice realizes the fields at discrete values of time, it is most convenient to perturb the metric, $h_{i j}$, in a synchronous gauge

$$
d s^{2}=d t^{2}-a^{2}(t)\left[\delta_{i j}+h_{i j}\right] d x^{i} d x^{j} .
$$

Additionally, the radiative part of $h_{i j}$ obeys the transverse-traceless conditions

$$
h_{i}^{i}=0 \text { and } h_{i j, j}=0 .
$$

The radiative perturbations obey sourced Klein-Gordon equations

$$
\ddot{h}_{i j}+3 H \dot{h}_{i j}-\frac{1}{a^{2}} \nabla^{2} h_{i j}=\frac{16 \pi}{m_{\mathrm{pl}}^{2}} S_{i j}^{T T},
$$

where the source term, $S_{i j}^{T T}$ is the transverse-traceless projection of the anisotropic stress tensor,

$$
S_{i j}=T_{i j}-\frac{\eta_{i j}}{3} T \text {. }
$$

We specify our model Eq. (7) and allow LATTICEEASY to evolve the fields and the scale factor. We can then calculate the source term of Eq. (20), and evolve the six metric perturbations, $h_{i j}^{T T}$. We can always check our numerical stability by checking to see if the metric perturbations are still transverse-traceless; transverse-traceless metric perturbations require both a transverse-traceless source and accurate evolution.

At any point during the simulation, we can calculate the power spectrum of gravitational radiation. The stress-energy associated with metric perturbations is [18],

$$
T_{\mu \nu}^{\mathrm{gw}}=\frac{1}{32 \pi}\left\langle h_{i j, \mu} h_{, \nu}^{i j}\right\rangle
$$

where the brackets denote a spatial average over at least a few wavelengths. The 00 component is the energy density,

$$
\rho_{\mathrm{gw}}=\frac{t^{\mu} t^{\nu}}{32 \pi}\left\langle h_{i j, \mu} h_{, \nu}^{i j}\right\rangle=\frac{1}{32 \pi} \sum_{i, j}\left\langle\dot{h}_{i j}^{2}\right\rangle,
$$

where $t^{\mu}=(1,0,0,0)$. Finally, we can invoke Parseval's theorem (see [17]) to rewrite Eq. (23) as

$$
\rho_{\mathrm{gw}}=\frac{1}{32 \pi} \frac{1}{V} \sum_{i, j} \int d^{3} \mathbf{k}\left|\dot{h}_{i j}(t, \mathbf{k})\right|^{2},
$$

where $V$ is the comoving volume over which the spatial average is being performed. We can then write

$$
\frac{d \rho_{\mathrm{gw}}}{d \ln k}=\frac{k^{3}}{32 \pi} \frac{1}{V} \sum_{i, j} \int d \Omega\left|\dot{h}_{i j}^{\mathrm{TT}}(\eta, \mathbf{k})\right|^{2},
$$

which can be transferred to present-day amplitude and frequency by $[17,19]$,

$$
\Omega_{\mathrm{gw}, 0} h^{2}=\Omega_{\mathrm{rad}, 0} h^{2}\left(\frac{g_{0}}{g_{e}}\right)^{1 / 3} \frac{1}{\rho_{\mathrm{tot}, \mathrm{e}}} \frac{d \rho_{\mathrm{gw}, \mathrm{e}}}{d \ln k},
$$

where the 0 and $e$ subscripts denote quantities defined today and the end of our simulations, respectively. We also keep the convention that $h$ absorbs the uncertainty in the present value of the Hubble parameter, $\Omega_{\mathrm{rad}, 0}$ is the current fraction of the energy density in the form of radiation, and $\rho_{\text {tot,e }}$ is the total energy density at the end of our simulations. The ratio, $g_{0} / g_{\mathrm{e}}$, is the number of degrees of freedom today to the number of degrees of freedom at matter/radiation equality. We approximate $g_{0} / g_{\mathrm{e}}=1 / 100$.

\section{RESULTS}

The first major difference between the structure of the gravitational-wave spectrum from self-ordering and that predicted by inflation is the lack of power at highfrequencies. This cut-off feature exists because we only considering larger then Hubble length fluctuations as sources of gravitational waves as there will be no/short lived gradient terms to source the gravitational waves inside the Hubble volumes by second order phase transitions.

We see in [20] that the cut-off frequency is related to the Hubble length at the time when the gravitational wave is generated

$$
f_{\text {peak }}=6 \times 10^{-10} \frac{k}{\sqrt{m_{\mathrm{pl}} H}} \mathrm{~Hz} .
$$

This, along with and the first Friedmann equation,

$$
H_{c}=\sqrt{\frac{8 \pi}{3}} \frac{\sqrt{\rho_{c}}}{m_{\mathrm{pl}}},
$$




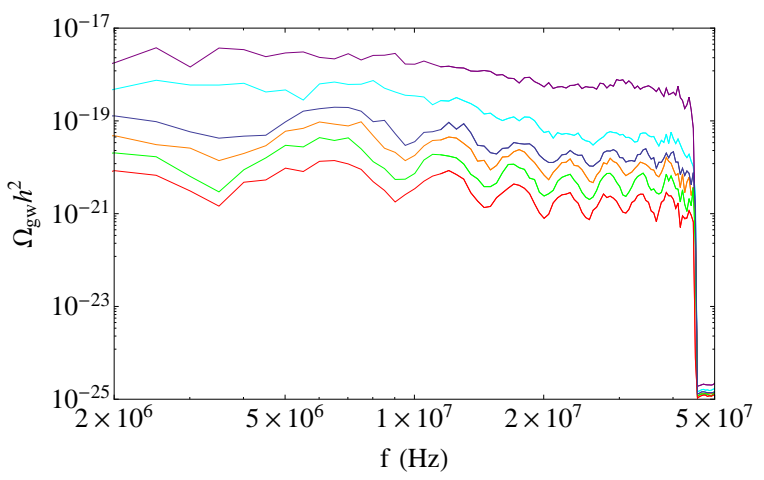

FIG. 1: The present-day gravitational wave spectrum from self-ordering. From top to bottom, $\mathcal{N}=2,3,4,5,8,16$.

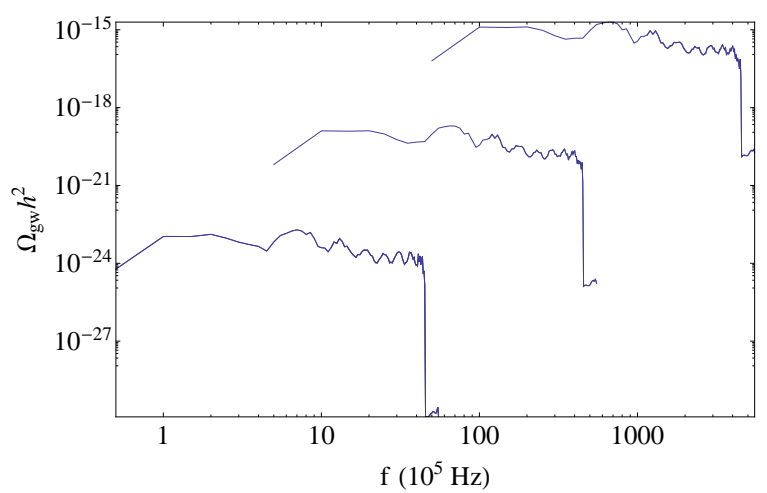

FIG. 2: The present-day gravitational wave spectrum from self-ordering. From top (rightmost) to bottom (leftmost), $\rho_{c}^{1 / 4}=10^{-3} m_{\mathrm{pl}}, \rho_{c}^{1 / 4}=10^{-4} m_{\mathrm{pl}}, \rho_{c}^{1 / 4}=10^{-5} m_{\mathrm{pl}}$.

allows us to determine where the cut-off should appear

$$
f_{\text {peak }} \sim 10^{11} \frac{\rho_{c}^{1 / 4}}{m_{\mathrm{pl}}} \mathrm{Hz}
$$

For example, with $\rho_{c}^{1 / 4}=10^{-4} m_{\mathrm{pl}}$ we expect the cutoff to be at $f=10^{7} \mathrm{~Hz}$ which agrees with the results of our simulation, shown in Fig. 1. The characteristic amplitude and scale invariant nature of the spectrum was expected from the analytical approach of $[6,21]$ and [7]. It is interesting to note that the spectrum is scale invariant for all the cases in Fig. 1, including those for $\mathcal{N}=2,3$; the analytical methods employed by [7] assumed that $\mathcal{N}$ is large.

In Fig. 2 we observe two important scaling effects. First, we recover the fact that the high-frequency cutoff predicted by Eq. 29 scales with $\rho^{1 / 4}$. We also see that the amplitude of the signal is proportional to the energy density of the Universe at the time of the transition,

$$
\Omega_{\mathrm{gw}} h^{2} \propto \rho_{c}
$$

This scaling is also suggested predicted in $[6,21]$ and [7].

A final step is to compare the numerical results here with the analytic arguments of $[6,21]$ and [7]. In both cases the authors use the model presented here to estimate the gravitational wave signal from reordering. The two sets of authors use slightly different parameterizations of the model; however, all authors arrive at the conclusion that there should be a scale-invariant gravitational wave spectrum from this transition

In $[6,21]$, the authors estimate the power in gravitational waves from field reordering to be (Eq. (10) in [21])

$$
\Omega_{\mathrm{gw}}^{\mathrm{JKM}}=\frac{1}{12 \pi^{3} H_{0}^{2}} k^{2} P\left(k, \tau_{0}\right),
$$

where $P(k, \tau)$ is calculated numerically, the Hubble constant today is $H, \tau_{0}$ is conformal time today and we've modified the normalization constant $1 / 12 \pi^{3}$ to reflect a convention choice in [6] and a typographical error in [21]. The strain power, $P$, ends up being a function only of $k \tau$. It peaks around $k \tau \approx 3.7$, although this corresponds to modes that entered the horizon during matter domination. To ensure that we're identifying modes that entered during radiation domination, we choose a mode, $k \approx 10 / \tau_{\text {eq }}$,

$$
P\left(\frac{k}{\tau_{\text {eq }}}, \tau_{0}\right)=\Omega_{\text {rad }} P\left(\frac{k}{\tau_{\text {eq }}}, \tau_{\text {eq }}\right),
$$

where we note that gravitational waves scale as a constant fraction of $\Omega_{\text {rad }}$ after matter-radiation equality, the subscript eq indicates evaluating quantities at the time of radiation-matter equality. We can read off $P\left(k / \tau_{\text {eq }}, \tau_{\text {eq }}\right)$ from Fig. 1 in [6],

$$
P\left(\frac{k}{\tau_{\mathrm{eq}}}, \tau_{0}\right) \approx 1000 \Omega_{\mathrm{rad}}
$$

So we can estimate the total gravitational wave energy per octave,

$$
\Omega_{\mathrm{gw}}^{\mathrm{JKM}}=\frac{1}{12 \pi^{3} H_{0}^{2}} \frac{10^{2}}{\tau_{\mathrm{eq}}^{2}} a_{\mathrm{eq}}^{2} P\left(\frac{3.7}{\tau_{\mathrm{eq}}}, \tau_{\mathrm{eq}}\right),
$$

where the physical wavevector now is $1 / a_{e q}$ larger than the physical wavevector at the time of radiation-matter equality. We can make a crude estimate of the value of conformal time at radiation-matter equality,

$$
\tau_{\mathrm{eq}}=\frac{1}{H_{\mathrm{eq}}} \int_{0}^{a_{\mathrm{eq}}} d a^{\prime}\left(\Omega_{R}+\Omega_{M} a+\Omega_{\Lambda} a^{4}\right)^{-1 / 2} \sim \frac{1}{50 H_{0}},
$$

where the fractional energy densities come from [3]. Putting Eq. (31) together with Eqs. $(33,34)$, we get an estimate,

$$
\Omega_{\mathrm{gw}}^{\mathrm{JKM}} h^{2}=\frac{99}{\mathcal{N}} \Omega_{\mathrm{rad}} h^{2}\left(\frac{v^{4}}{4 \mathcal{N} m_{\mathrm{pl}}^{4}}\right)
$$

or, imposing our parameterization of the current-day Hubble constant, setting $\Omega_{\mathrm{rad}} h^{2} \approx 2 \times 10^{-5}$ and using Eqs. (14,17)

$$
\Omega_{\mathrm{gw}}^{\mathrm{JKM}} h^{2}=\frac{0.016}{\mathcal{N}} \frac{\alpha}{\lambda} \frac{\rho_{c}}{m_{\mathrm{pl}}^{4}} .
$$


In [7], the authors predict a scale-invariant power spectrum (Eq. (5.2) of [7])

$$
\Omega_{\mathrm{gw}}^{\mathrm{FFDG}} h^{2} \simeq \frac{511}{\mathcal{N}} \Omega_{\mathrm{rad}} h^{2}\left(\frac{v}{\sqrt{2} m_{\mathrm{pl}}}\right)^{4}
$$

where we use our definition of $v$ and our parameterization $\Omega_{g w} h^{2}$. Using Eqs. $(14,17)$ along with $\Omega_{\mathrm{rad}} h^{2} \approx 2 \times 10^{-5}$, the expression in Eq. (37) reduces to

$$
\Omega_{\mathrm{gw}}^{\mathrm{FFDG}} h^{2} \simeq \frac{0.082}{\mathcal{N}} \frac{\alpha}{\lambda} \frac{\rho_{c}}{m_{\mathrm{pl}}^{4}} .
$$

These two estimates should vary from our simulations by one important factor. In both cases, the Universe is comprised only of the scalar fields. To preserve a radiation-dominated phase during and after the phase transition, we have, inherently, diluted the source by a factor of $\alpha$ which dilutes the analytic estimates Eqs. $(35,38)$ by a factor of $\alpha^{2}$.

It is worth pointing out that some of the phase transitions we have simulated result in the production of global topological defects. Specifically, global strings for $\mathcal{N}=2$, global monopoles $\mathcal{N}=3$, and global textures for $\mathcal{N}>3$. Surprisingly, we find that the gravitational radiation produced is consistent with the large $\mathcal{N}$ approximation even for low values of $\mathcal{N}$, where the approximation is not valid (see our analytic estimates above). The gravitational wave backgrounds produced by global strings and monopoles are larger than those produced by textures. We will investigate these cases in more detail in a future publication.

\section{DISCUSSION}

Phase transitions at high energies are a generic consequence of (almost) all models of high energy physics. Since there is no unique model of physics at this scale, we are forced to look for generic observational consequences at this scale. Second order phase transitions will not produce gravitational radiation over one Hubble time, yet the reordering of the fields that mediate the transition could produce characteristic gravitational radiation over a wide range of scales. Although this signature could be misinterpreted as the gravitational radiation from primordial quantum fluctuations, it might be possible to distinguish the two at very high frequencies. Such a highfrequency detection would also carry information about the energy scale at which the phase transition occurred.

We considered the phenomenological model of $[6,21]$ and [7] in which an $\mathcal{O}(\mathcal{N})$ symmetric false vacuum is dynamically broken into a $\mathcal{O}(\mathcal{N}-1)$ true vacuum, we find this produces a scale invariant gravitational wave spectrum whose amplitude depends inversely on number of fields. These results are summarized in Table I. Our numerical results suggest that the large $\mathcal{N}$ is not needed to make a scale invariant spectrum. Since the results of
TABLE I: Spectral amplitudes as a function of number of fields for simulations with $\left(\rho_{c}^{1 / 4}=10^{-4} m_{p l}, \alpha=\lambda=0.1\right)$. The numerical values, $\Omega_{\mathrm{gw}} h^{2}$, are an average value taken from the simulations, while the values in the second two columns are obtained from Eq. (35) or Eq. (38).

\begin{tabular}{cccc}
\hline \hline $\mathcal{N}$ & $\Omega_{\mathrm{gw}} h^{2}$ & $\alpha^{2} \Omega_{\mathrm{gw}}^{\mathrm{JKM}} h^{2}$ & $\alpha^{2} \Omega_{\mathrm{gw}}^{\mathrm{FFG}} h^{2}$ \\
\hline 2 & $1.0 \times 10^{-18}$ & $9.0 \times 10^{-21}$ & $4.1 \times 10^{-20}$ \\
4 & $3.8 \times 10^{-20}$ & $4.0 \times 10^{-21}$ & $2.1 \times 10^{-20}$ \\
8 & $8.3 \times 10^{-21}$ & $2.0 \times 10^{-21}$ & $1.0 \times 10^{-20}$ \\
16 & $3.1 \times 10^{-21}$ & $1.0 \times 10^{-21}$ & $5.1 \times 10^{-21}$
\end{tabular}

TABLE II: Spectral amplitudes as a function of $\rho_{c}$ simulations with $(\mathcal{N}=4, \alpha=\lambda=0.1)$. The numerical values, $\Omega_{\mathrm{gw}} h^{2}$, are an average value taken from the simulations, while the values in the second two columns are obtained from Eq. (35) or Eq. (38).

\begin{tabular}{cccc}
\hline \hline$\rho_{c}^{1 / 4}\left(m_{p l}\right)$ & $\Omega_{\mathrm{gw}} h^{2}$ & $\alpha^{2} \Omega_{\mathrm{gw}}^{\mathrm{JKM}} h^{2}$ & $\alpha^{2} \Omega_{\mathrm{gw}}^{\mathrm{FFG}} h^{2}$ \\
\hline $10^{-3}$ & $4.7 \times 10^{-16}$ & $4.0 \times 10^{-17}$ & $2.1 \times 10^{-16}$ \\
$10^{-4}$ & $3.8 \times 10^{-20}$ & $4.0 \times 10^{-21}$ & $2.1 \times 10^{-20}$ \\
$10^{-5}$ & $4.0 \times 10^{-24}$ & $4.0 \times 10^{-25}$ & $2.1 \times 10^{-24}$
\end{tabular}

$[6,7,21]$ are derived using a large $\mathcal{N}$ approximation for the amplitude, one does not expect these estimates to be a perfect estimator of the amplitude of the gravitational waves in the low- $\mathcal{N}$ limit. Additionally, the results for varying values of $\rho_{c}$ are given in Table II. We find that our simulations differ from analytic estimates by only a small amount and are more consistent at large $\mathcal{N}$, consistent with the fact that analytic methods are derived from a large $\mathcal{N}$ expansion.

\section{ACKNOWLEDGMENTS}

We thank Latham Boyle, Andrew Tolley, Harsh Mathur and Katherine Jones-Smith for useful discussions. JTG is supported by the National Science Foundation, PHY-1068080, and a Cottrell College Science Award from the Research Corporation. The work of $\mathrm{XS}$ and BV is supported by National Science Foundation grants PHY-0970074, PHY-0955929, and PHY-0758155, and the University of Wisconsin-Milwaukee Research Growth Initiative. Research at the Perimeter Institute for Theoretical Physics is supported by the Government of Canada through Industry Canada and by the Province of Ontario through the Ministry of Research \& Innovation. JTG would also like to thank the University of Wisconsin-Milwaukee for its generous hospitality while some of this research was completed. LRP would like to thank the Perimeter Institute for Theoretical Physics for their hospitality while some of this work was being completed there. 


\section{Appendix A: Thermal Initial Conditions}

The probability of the scalar field taking some average value, $\bar{\phi}$, is given by

$$
P\left(\phi_{v}=\bar{\phi}\right)=\int D \phi \mathcal{P}[\phi] \delta\left(\phi_{v}-\bar{\phi}\right)=\left\langle\delta\left(\phi_{v}-\bar{\phi}\right)\right\rangle
$$

where $\mathcal{P}[\phi]$ is the probability functional and we've introduced the volume-averaged field

$$
\begin{aligned}
\phi_{v} & =\frac{1}{V} \int_{V} d^{3} x \phi(x) \\
& =\frac{1}{V} \int_{-\infty}^{\infty} d^{3} x I(x) \phi(x),
\end{aligned}
$$

where $I(x)$ is a window function introduced for later computational convenience. We compute Eq. (A1) using a Gaussian approximation

$$
P\left(\phi_{v}=\bar{\phi}\right) \approx \sqrt{\frac{1}{2 \pi \sigma^{2}}} \exp \left(-\frac{(\bar{\phi}-\mu)^{2}}{2 \sigma^{2}}\right),
$$

with $\mu=\left\langle\phi_{v}\right\rangle$ and $\sigma^{2}=\left\langle\phi_{v}^{2}\right\rangle-\left\langle\phi_{v}\right\rangle^{2}$. The problem is now to compute the moments of the field

$$
\begin{aligned}
\left\langle\phi_{v}\right\rangle & =\int D \phi \mathcal{P}[\phi]\left(\frac{1}{V} \int_{V} d^{3} x \phi(x)\right) \\
\left\langle\phi_{v}^{2}\right\rangle & =\int D \phi \mathcal{P}[\phi]\left(\frac{1}{V^{2}} \int_{V} d^{3} x d^{3} y \phi(x) \phi(y)\right)
\end{aligned}
$$

The full probability functional can be found in, e.g. [22]. Here we consider only the leading order temperature dependence, which modifies the potential by replacing $m$ with a temperature dependent term, $m_{\mathrm{eff}}(\beta)$, where $\beta \propto T^{-1}$. Choosing a temperature above $m_{\mathrm{eff}}=0$ puts us in a symmetric phase of the effective potential. We then have

$$
\begin{aligned}
\mathcal{P}[\phi] & \approx \frac{1}{Z} \exp \left(-\beta H_{\mathrm{eff}}[\phi]\right) \\
& =\frac{1}{Z} \exp \left(\frac{-\beta}{2} \int d^{3} x \phi(x)\left(\nabla+m_{\mathrm{eff}}(\beta)^{2}\right) \phi(x)\right) .
\end{aligned}
$$

Henceforth we write $m_{\mathrm{eff}}=m_{\mathrm{eff}}(\beta)$. Defining

$$
\begin{aligned}
W[J]=\int D \phi \exp \left(\frac{-\beta}{2} \int d^{3} x \phi(x)\left(\nabla+m_{\mathrm{eff}}^{2}\right) \phi(x)\right. \\
+J(x) \phi(x)) \\
=Z \exp \left(\frac{1}{2 \beta} \iint d^{3} x d^{3} y J(x) K^{-1}(x-y) J(y)\right),
\end{aligned}
$$

with $\left(-\nabla_{x}^{2}+m_{\text {eff }}^{2}\right) K^{-1}(x-y)=\delta^{3}(x-y)$ allows us to write the moments of the field as

$$
\begin{aligned}
\left\langle\phi_{v}\right\rangle= & \left.\frac{1}{V} \int_{-\infty}^{\infty} d^{3} x I(x) \frac{\delta}{\delta J(x)} W[J]\right|_{J=0} \\
\left\langle\phi_{v}^{2}\right\rangle= & \frac{1}{V} \int_{-\infty}^{\infty} d^{3} x d^{3} y I(x) I(y) \\
& \times\left.\frac{\delta}{\delta J(x)} \frac{\delta}{\delta J(y)} W[J]\right|_{J=0} .
\end{aligned}
$$

Choosing a Gaussian window such that $\int d^{3} x I(x)=$ $4 \pi R^{3} / 3$ and evaluating the integrals leads to

$$
\begin{aligned}
\left\langle\phi_{v}\right\rangle & =0 \\
\left\langle\phi_{v}^{2}\right\rangle & =\frac{1}{4 \pi^{3 / 2} \beta R}-\frac{m_{\mathrm{eff}} \exp \left(m_{\mathrm{eff}}^{2} R^{2}\right)}{4 \pi \beta R} \operatorname{erfc}\left(m_{\mathrm{eff}} R\right) .
\end{aligned}
$$

Putting this all together we have

$$
P\left(\phi_{v}=\bar{\phi}\right)=\sqrt{\frac{H_{0} T_{0}}{8 \pi^{5 / 2}}} \exp \left(-\frac{\bar{\phi}^{2}}{4 \pi^{3 / 2}} H_{0} T_{0}\right) .
$$

[1] A. A. Starobinsky, JETP Lett. 30, 682 (1979) [Pisma Zh. Eksp. Teor. Fiz. 30, 719 (1979)].

[2] V. A. Rubakov, M. V. Sazhin and A. V. Veryaskin, Phys. Lett. B 115, 189 (1982).

[3] E. Komatsu et al. [WMAP Collaboration], Astrophys. J. Suppl. 192, 18 (2011) [arXiv:1001.4538 [astro-ph.CO]].

[4] [Planck Collaboration], arXiv:astro-ph/0604069.

[5] L. M. Krauss, Phys. Lett. B 284, 229 (1992).

[6] K. Jones-Smith, L. M. Krauss and H. Mathur, Phys. Rev. Lett. 100, 131302 (2008) [arXiv:0712.0778 [astro-ph]].

[7] E. Fenu, D. G. Figueroa, R. Durrer and J. GarciaBellido, JCAP 0910, 005 (2009) [arXiv:0908.0425 [astro-ph.CO]].

[8] E. Witten, Phys. Rev. D 30, 272 (1984).
[9] A. Kosowsky, M. S. Turner and R. Watkins, Phys. Rev. D 45, 4514 (1992).

[10] M. Kamionkowski, A. Kosowsky, M. S. Turner, Phys. Rev. D49, 2837-2851 (1994). [astro-ph/9310044].

[11] H. C. Child and J. T. Giblin, Jr. (to appear)

[12] G. N. Felder and I. Tkachev, [arXiv:hep-ph/0011159].

[13] A. V. Frolov, JCAP 0811, 009 (2008). [arXiv: 0809.4904 [hep-ph]].

[14] R. Easther, H. Finkel, N. Roth, JCAP 1010, 025 (2010). [arXiv: 1005.1921 [astro-ph.CO]].

[15] Z. Huang, Phys. Rev. D83, 123509 (2011). [arXiv:1102. 0227 [astro-ph.CO]].

[16] R. Easther, J. T. Giblin, Jr and E. A. Lim, Phys. Rev. Lett. 99, 221301 (2007) [arXiv:astro-ph/0612294]. 
[17] R. Easther, J. T. Giblin, Jr and E. A. Lim, Phys. Rev. D 77, 103519 (2008) [arXiv:0712.2991 [astro-ph]].

[18] C. W. Misner, K. S. Thorne, J. A. Wheeler, San Francisco 1973, 1279p.

[19] L. R. Price and X. Siemens, Phys. Rev. D 78, 063541 (2008) [arXiv:0805.3570 [astro-ph]].

[20] R. Easther, E. A. Lim, JCAP 0604, 010 (2006). [astro-ph/0601617].

[21] L. M. Krauss, K. Jones-Smith, H. Mathur and J. Dent, Phys. Rev. D 82, 044001 (2010) [arXiv:1003.1735 [astro-ph.CO]].

[22] M. Hindmarsh, R. J. Rivers, Nucl. Phys. B417, 506-526 (1994). [hep-ph/9307253]. 